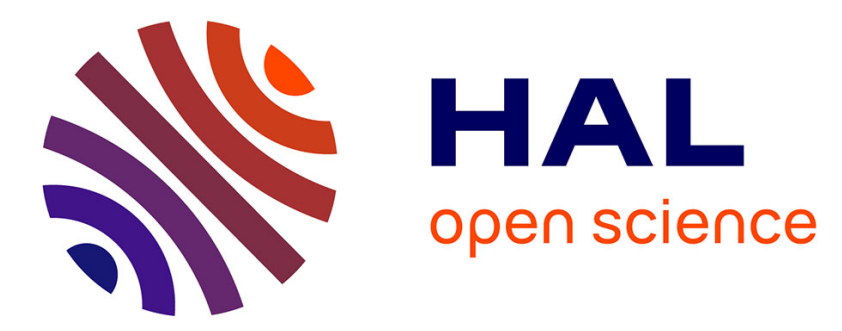

\title{
A simple pressure stabilization method for the Stokes equation
}

\author{
Roland Becker, Peter Hansbo
}

\section{To cite this version:}

Roland Becker, Peter Hansbo. A simple pressure stabilization method for the Stokes equation. International Journal for Numerical Methods in Biomedical Engineering, 2008, 24 (11), pp.1421-1430. 10.1002/cnm.1041 . hal-01352889

\section{HAL Id: hal-01352889 \\ https://hal.science/hal-01352889}

Submitted on 10 Aug 2016

HAL is a multi-disciplinary open access archive for the deposit and dissemination of scientific research documents, whether they are published or not. The documents may come from teaching and research institutions in France or abroad, or from public or private research centers.
L'archive ouverte pluridisciplinaire HAL, est destinée au dépôt et à la diffusion de documents scientifiques de niveau recherche, publiés ou non, émanant des établissements d'enseignement et de recherche français ou étrangers, des laboratoires publics ou privés. 


\title{
A simple pressure stabilization method for the Stokes equation
}

\author{
Roland Becker ${ }^{1}$ and Peter Hansbo ${ }^{2}$ \\ ${ }^{1}$ Laboratoire de Mathématiques Appliquées, Université de Pau et des Pays de l'Adour, BP 1155, \\ 64013 PAU Cedex, France \\ ${ }^{2}$ Division of Computational Mathematics, Chalmers University of Technology and Göteborg University, \\ S-412 96 Göteborg, Sweden
}

In this paper, we consider a stabilization method for the Stokes problem, using equal-order interpolation of the pressure and velocity, which avoids the use of the mesh size parameter in the stabilization term. We show that our approach is stable for equal-order interpolation in the case of piecewise linear and piecewise quadratic polynomials on triangles. In the case of linear polynomials, we retrieve a well-known idea of using mass lumping as a stabilization mechanism.

\section{INTRODUCTION}

Discretization of the Stokes equations requires special care since a stable approximation of pressure places constraints on the coupling with velocities, see [1]. Besides the construction of stable pairs of subspaces, finite element stabilization schemes are successfully used in practice. The idea is to use standard continuous finite element spaces of equal degree for both pressure and velocities. The lack of stability of the discrete gradient operator between these spaces is compensated by addition of appropriate stabilization terms.

One of the first stabilization methods was proposed by Brezzi and Pitkäranta [2], who added a weighted Laplace operator on the pressure space, which yields an optimally convergent scheme 
for equal-order $P^{1}$-approximations. In [3], a weighted least-squares formulation which also works on quadrilaterals and higher-order finite elements is presented.

A drawback of the least-squares formulation is the pressure-velocity couplings and the difficulty to formulate explicit time-stepping methods in the time-dependent case. Alternative formulations have been developed in [4] where instead the pressure gradient is introduced as an additional variable (three-field formulation) [5], where a stabilization based on local projections between two different spaces is used, and [6] where the jump of the normal derivative over element edges is used as stabilization. A generalization of the method in [2] was suggested in [7] by using of polynomial projections onto a space of polynomials of one degree less than that used in the approximation.

The main idea of the discretization proposed here is to use the difference between a consistent mass matrix $\mathbf{M}$ and a underintegrated mass matrix $\tilde{\mathbf{M}}$ as stabilization term for pressure:

$$
s\left(p_{h}, q_{h}\right):=\mathbf{q}^{\mathrm{T}}(\tilde{\mathbf{M}}-\mathbf{M}) \mathbf{p}
$$

where we denote by $\mathbf{p}=\left(p_{i}\right)_{i=1}^{n} \in \mathbb{R}^{N}$ the coefficients of the discrete pressure $p_{h} \in Q_{h}$ in the Lagrange basis $\psi_{i}$ of the pressure space: $p_{h}=\sum_{i=1}^{N} p_{i} \psi_{i}$. The pressure matrix is in general already available in finite element flow solvers, since it is often used as a preconditioner for the Schur complement of the coupled system. Therefore, stabilization (1) requires only minor modification of coding and only few additional computations. Indeed, in the case of a piecewise linear pressure approximation, matrix $\tilde{\mathbf{M}}$ is a diagonal matrix, known as the lumped mass matrix, having the row sum of $\mathbf{M}$ on the diagonal. Thus, in this case, computing $\mathbf{M}$ also gives $\tilde{\mathbf{M}}$. In this context, we remark that the idea of using $\tilde{\mathbf{M}}-\mathbf{M}$ as a stabilization mechanism for piecewise linear finite element methods is well known and has been used, e.g. by Löhner et al. [8], for the numerical solution of compressible flow.

In this article we present some basic results concerning the case of standard $P^{1}$ and $P^{2}$ finite elements.

We denote by $V_{h} \subset H_{0}^{1}(\Omega)=: V$ the discrete velocity space and by $Q_{h} \subset L^{2}(\Omega) \backslash \mathbb{R}=: Q$ the discrete pressure space, based on a shape-regular affine triangulation $\mathscr{T}_{h}$ of the bounded polygonal domain $\Omega \subset \mathbb{R}^{n}, n=2,3$. We suppose that both $V_{h}$ and $Q_{h}$ consist of continuous piecewise linear $\left(P^{1}\right)$ or continuous piecewise quadratic finite element functions $\left(P^{2}\right)$.

We denote the Lagrange interpolation operator on the space $P^{r}, r=1,2,3$ by $I_{h}^{r}: C(\bar{\Omega}) \rightarrow Q_{h}$.

Further, we denote the bilinear form describing the Stokes equations by $a:(V \times Q) \times(V \times Q) \rightarrow \mathbb{R}$ :

$$
a((v, p),(w, q)):=(\nabla v, \nabla w)-(p, \operatorname{div} w)+(\operatorname{div} v, q)
$$

For given $f \in V^{*}$, the standard weak formulation of the Stokes equations reads: Find $(v, p) \in V \times Q$ such that

$$
a((v, p),(w, q))=(f, v) \quad \forall(w, q) \in V \times Q
$$

The discrete solution is defined by: Find $\left(v_{h}, p_{h}\right) \in V_{h} \times Q_{h}$ such that

$$
a\left(\left(v_{h}, p_{h}\right),\left(w_{h}, q_{h}\right)\right)+\alpha s\left(p_{h}, q_{h}\right)=\left(f, v_{h}\right) \quad \forall\left(w_{h}, q_{h}\right) \in V_{h} \times Q_{h}
$$

It follows from the stability result below that there exists a unique solution. We will analyse two cases: in the first one we use continuous $P^{1}$ finite elements for velocity and pressure, in the second case we use quadratics for both unknowns. 


\section{Remark 1}

In a recent paper by $\mathrm{Li}$ and $\mathrm{He}$ [9], a similar stabilization method has been proposed. An important distinction is that in [9] only equal-order $P^{1}$ and $Q^{1}$ interpolations were used, using the difference between the mass matrix and a one-point under-integrated mass matrix for stabilization. This allows for a direct analogy with the method of Dohrmann and Bochev [7]; indeed the methods of $[7,9]$ are identical from a numerical point of view for these low-order approximations. In the approach adopted in this paper, there is no such equivalence.

\section{THE CASE OF $P^{1}$-APPROXIMATIONS}

We start with some remarks on the stabilization bilinear form (1)

\section{Lemma 1}

We can rewrite stabilization (1) as

$$
s(p, q):=\int_{\Omega}\left(I_{h}^{1}(p q)-p q\right) \mathrm{d} x
$$

Proof

This follows from the fact that $p_{i}=p\left(x_{i}\right)$ :

$$
\begin{aligned}
\mathbf{q}^{\mathrm{T}}(\tilde{\mathbf{M}}-\mathbf{M}) \mathbf{p} & =\sum_{i j} q_{i}\left(\delta_{i j} \sum_{k} M_{i k}-M_{i j}\right) p_{j} \\
& =\int_{\Omega} q_{i} \psi_{i} p_{i} \mathrm{~d} x-\int_{\Omega} q p \mathrm{~d} x
\end{aligned}
$$

From Lemma 1 we obtain the following result.

\section{Proposition 1}

There exists a constant $c$ such that for $p_{h} \in Q_{h}$

$$
\sum_{K \in \mathscr{T}_{h}} h_{K}^{2}\left\|\nabla p_{h}\right\|^{2} \leqslant c s\left(p_{h}, p_{h}\right)
$$

Proof

It is sufficient to proof (6) on the reference element $\hat{K}$. Next we observe that for a linear function $\hat{p}$ on $\hat{K}$

$$
I_{h}^{1} \hat{p}^{2}(x) \geqslant \hat{p}^{2}(x) \quad \forall x \in K
$$

and equality only holds in case $\hat{p}$ is constant. Indeed the polynomial $g(x):=I_{h}^{1} \hat{p}^{2}(x)-\hat{p}^{2}(x)$ has Hessian $\nabla^{2} g(x)=-\nabla p(x) \otimes \nabla p(x)$, which is negative definite unless $\nabla p(x)=0$.

It follows that $\int_{\hat{K}}\left\{I_{h}^{1}(\hat{p} \hat{q})-\hat{p} \hat{q}\right\} \mathrm{d} \hat{x}$ and $\int_{\hat{K}} \hat{\nabla} \hat{p} \cdot \hat{\nabla} \hat{q} \mathrm{~d} \hat{x}$ are equivalent symmetric bilinear forms on the reference element and (6) follows from scaling. 
It is well known (cf. [1]) that there is a $\gamma>0$ so that for $p \in Q$ there exists $u \in V$ such that

$$
(p, \operatorname{div} u) \geqslant \gamma^{2}\|p\|^{2} \quad \text { and } \quad\|\nabla u\| \leqslant\|p\|
$$

In order to simplify notations, we introduce the norm

$$
\|(v, p)\|^{2}=\|p\|^{2}+\|\nabla v\|^{2}+s(p, p)^{2}
$$

Theorem 2

Let $\alpha$ be big enough. Then there exists $\Gamma>0$ such that for given $\left(v_{h}, p_{h}\right) \in V_{h} \times Q_{h}$ there exists $\left(w_{h}, q_{h}\right) \in V_{h} \times Q_{h}$ with $\left\|\left(w_{h}, q_{h}\right)\right\| \leqslant 1$ and

$$
a\left(\left(v_{h}, p_{h}\right),\left(w_{h}, q_{h}\right)\right)+\alpha s\left(p_{h}, q_{h}\right) \geqslant \Gamma\left\|\left|\left(v_{h}, p_{h}\right) \|\right|\right.
$$

Proof

There exists $u \in V$ such that for $p_{h} \in Q_{h}$ given, (7) holds. Let $u_{h}$ be the Clément interpolation (cf. [10]) of $u$. Integration by parts gives

$$
\begin{aligned}
\left(p_{h}, \operatorname{div}\left(u-u_{h}\right)\right) & =-\left(\nabla p_{h}, u-u_{h}\right) \\
& \leqslant\left(\sum_{K} h_{K}^{2}\left\|\nabla p_{h}\right\|^{2}\right)^{1 / 2}\left(\sum_{K} h_{K}^{-2}\left\|u-u_{h}\right\|^{2}\right)^{1 / 2} \\
& \leqslant C s\left(p_{h}, p_{h}\right)^{1 / 2}\|\nabla u\|
\end{aligned}
$$

by (6) and the interpolation property of $u_{h}$.

Therefore we have, using the stability of the Clément operator,

$$
\begin{aligned}
a\left(\left(v_{h}, p_{h}\right),\left(-u_{h}, 0\right)\right) & =-\left(\nabla v_{h}, \nabla u_{h}\right)+\left(p_{h}, \operatorname{div} u_{h}\right) \\
& \geqslant-\left\|\nabla v_{h}\right\|\left\|\nabla u_{h}\right\|+\left(p_{h}, \operatorname{div} u\right)+\left(p_{h}, \operatorname{div}\left(u_{h}-u\right)\right) \\
& \geqslant \gamma^{2}\left\|p_{h}\right\|^{2}-C_{0}\left\|\nabla v_{h}\right\|\|\nabla u\|-C_{1} s\left(p_{h}, p_{h}\right)^{1 / 2}\|\nabla u\| \\
& \geqslant \frac{\gamma^{2}}{4}\left\|p_{h}\right\|^{2}-\frac{C_{2}}{\gamma^{2}}\left\|\nabla v_{h}\right\|^{2}-\frac{C_{3}}{\gamma^{2}} s\left(p_{h}, p_{h}\right)
\end{aligned}
$$

It follows that with $\theta$ sufficiently small we have $\left\|\left(v_{h}-\theta u_{h}, p_{h}\right)\right\| \leqslant C\left\|\left(v_{h}, p_{h}\right)\right\|$ and

$$
a\left(\left(v_{h}, p_{h}\right),\left(v_{h}-\theta u_{h}, p_{h}\right)\right) \geqslant C\left(\left\|p_{h}\right\|^{2}+s\left(p_{h}, p_{h}\right)+\left\|\nabla v_{h}\right\|^{2}\right)
$$

which concludes the proof.

Theorem 3

Let (9) hold. Then there is a constant $C$ such that

$$
\left\|\left(v-v_{h}, p-p_{h}\right)\right\| \leqslant C \inf _{\left(w_{h}, q_{h}\right) \in V_{h} \times Q_{h}}\left\|\nabla\left(v-w_{h}\right)\right\|+\left\|p-q_{h}\right\|+s\left(q_{h}, q_{h}\right)^{1 / 2}
$$


Especially, if $v \in H^{2}(\Omega)$ and $p \in H^{1}(\Omega)$ we have the standard error estimate:

$$
\left\|p-p_{h}\right\|+\left\|\nabla\left(v-v_{h}\right)\right\| \leqslant C h
$$

Proof

Let $\left(w_{h}, q_{h}\right) \in V_{h} \times Q_{h}$ be arbitrary. We have

$$
\begin{aligned}
\Gamma\left\|\left(v_{h}-w_{h}, p_{h}-q_{h}\right)\right\| \mid & \leqslant \sup _{\left(y_{h}, r_{h}\right) \in V_{h} \times Q_{h}} a\left(\left(v_{h}-w_{h}, p_{h}-q_{h}\right),\left(y_{h}, r_{h}\right)\right)+\alpha s\left(p_{h}-q_{h}, r_{h}\right) \\
& =\sup _{\left(y_{h}, r_{h}\right) \in V_{h} \times Q_{h}} a\left(\left(v-w_{h}, p-q_{h}\right),\left(y_{h}, r_{h}\right)\right)-\alpha s\left(q_{h}, r_{h}\right)
\end{aligned}
$$

since $(v, p)$ is the solution of (3), yielding (11).

In order to obtain (12), we set $q_{h}:=I_{h}^{1} p$ and $w_{h}:=I_{h}^{1} v$. We need to estimate the stabilization term:

$$
s\left(I_{h}^{1} p, I_{h}^{1} p\right)=\int_{K}\left\{I_{h}^{1}\left(\left(I_{h}^{1} p\right)^{2}\right)-\left(I_{h}^{1} p\right)^{2}\right\} \mathrm{d} x
$$

Let $\phi \in H^{2}(K)$. By means of the Bramble-Hilbert lemma, we obtain the following estimate:

$$
\int_{K}\left\{I_{h}^{1}(\phi)-\phi\right\} \mathrm{d} x \leqslant C h_{K}^{2} \int_{K}\left|\nabla^{2} \phi\right| \mathrm{d} x
$$

We apply this result to $\phi=\left(I_{h}^{1} p\right)^{2}$. Since $I_{h}^{1} p$ is a linear function we find that the Hessian

$$
\nabla^{2}\left(I_{h}^{1} p\right)^{2}=\nabla I_{h}^{1} p \otimes \nabla I_{h}^{1} p
$$

and finally

$$
s\left(I_{h}^{1} p, I_{h}^{1} p\right) \leqslant C h_{k}^{2}\left\|\nabla I_{h}^{1} p\right\|^{2}
$$

We conclude by stability of $I_{h}^{1}$ in $H^{1}$.

\section{THE CASE OF $P^{2}$-APPROXIMATIONS}

For the $P^{2}$ case, we can no longer rely on norm equivalence. The proof will instead be based on the fact that the combination of $P^{2}$ for the velocities and $P^{1}$ for the pressure is stable (the Taylor-Hood approximation, cf. [10]). The stabilization matrix will then be used to control the difference between the $P^{1}$ and $P^{2}$ spaces. We here define the stabilization term as

$$
s(p, q):=\int_{\Omega}\left(I_{h}^{3}(p q)-p q\right) \mathrm{d} x
$$

where $I_{h}^{3}$ is the Lagrange interpolation operator onto cubic polynomials. We have the following result. 


\section{Lemma 4}

Split the pressure into two parts, $p_{h}=p_{1}+p_{2}$, where $p_{1}$ is the piecewise linear part and $p_{2}$ is the remainder. Then there holds

$$
s\left(p_{h}, p_{h}\right) \geqslant c_{0}\left\|p_{2}\right\|^{2}
$$

\section{Proof}

We only need to check this property on a reference element since the mapping to the physical element is affine, and both sides of (13) are given as sums over the elements. Denote by $\mathbf{m}$ the matrix-valued function whose components are $m_{i j}:=\psi_{i} \psi_{j}$, where $\left\{\psi_{i}\right\}, i=1, \ldots, 6$ is the Lagrangian basis of second-degree polynomials. We now compute the element mass matrix $\mathbf{M}_{K}$ such that

$$
\mathbf{M}_{K}:=\int_{K} \mathbf{m} \mathrm{d} x
$$

and the interpolation onto the Lagrangian cubic basis $\left\{\varphi_{i}\right\}, i=1, \ldots, 10$, given by

$$
\tilde{\mathbf{M}}_{K}:=\sum_{k=1}^{10} \int_{K} \varphi_{k}(x) \mathbf{m}\left(x_{k}\right) \mathrm{d} x
$$

A simple computation shows that, on the unit element numbered in the order of corner nodes followed by edge nodes,

$$
\mathbf{M}_{K}=\left[\begin{array}{cccccc}
1 / 60 & -1 / 360 & -1 / 360 & 0 & -1 / 90 & 0 \\
-1 / 360 & 1 / 60 & -1 / 360 & 0 & 0 & -1 / 90 \\
-1 / 360 & -1 / 360 & 1 / 60 & -1 / 90 & 0 & 0 \\
0 & 0 & -1 / 90 & 4 / 45 & 2 / 45 & 2 / 45 \\
-1 / 90 & 0 & 0 & 2 / 45 & 4 / 45 & 2 / 45 \\
0 & -1 / 90 & 0 & 2 / 45 & 2 / 45 & 4 / 45
\end{array}\right]
$$

and

$$
\tilde{\mathbf{M}}_{K}=\left[\begin{array}{cccccc}
13 / 540 & 1 / 1080 & 1 / 1080 & -1 / 135 & -1 / 90 & -1 / 135 \\
1 / 1080 & 13 / 540 & 1 / 1080 & -1 / 135 & -1 / 135 & -1 / 90 \\
1 / 1080 & 1 / 1080 & 13 / 540 & -1 / 90 & -1 / 135 & -1 / 135 \\
-1 / 135 & -1 / 135 & -1 / 90 & 14 / 135 & 2 / 45 & 2 / 45 \\
-1 / 90 & -1 / 135 & -1 / 135 & 2 / 45 & 14 / 135 & 2 / 45 \\
-1 / 135 & -1 / 90 & -1 / 135 & 2 / 45 & 2 / 45 & 14 / 135
\end{array}\right]
$$

and the eigenvalues of $\tilde{\mathbf{M}}_{K}-\mathbf{M}_{K}$ are given by $\left\{0,0,0, \frac{1}{54}, \frac{1}{54}, \frac{4}{135}\right\}$, where the zero eigenvalues correspond to the squared linear terms in $\left\{\psi_{i}\right\}$ which are exactly integrated in both $\mathbf{M}_{K}$ and $\tilde{\mathbf{M}}_{K}$. The matrix $\tilde{\mathbf{M}}_{K}-\mathbf{M}_{K}$ is thus positive definite on the subspace consisting of the quadratic part of the basis $\left\{\psi_{i}\right\}$ and the result follows. 


\section{Lemma 5}

Let $\alpha$ be big enough. Then there exists $w_{h} \in V_{h}$ such that for given $p_{h} \in Q_{h}$ there holds

$$
\begin{aligned}
\left(p_{h}, \operatorname{div} w_{h}\right)+\alpha s\left(p_{h}, p_{h}\right) & \geqslant \gamma^{2}\left\|p_{h}\right\|^{2} \\
\left\|\nabla w_{h}\right\|^{2} & \leqslant C\left(\left\|p_{h}\right\|^{2}+s\left(p_{h}, p_{h}\right)\right)
\end{aligned}
$$

\section{Proof}

Split the pressure as in Lemma 4. It follows that $s\left(p_{h}, p_{h}\right)=s\left(p_{2}, p_{2}\right)$, and from the stability of the Taylor-Hood element we know that $\exists w_{h} \in V_{h}$ such that

$$
\begin{aligned}
\left(p_{1}, \operatorname{div} w_{h}\right) & \geqslant \gamma_{1}^{2}\left\|p_{1}\right\|^{2} \\
\left\|\nabla w_{h}\right\|^{2} & \leqslant\left\|p_{1}\right\|^{2} \leqslant\left\|p_{h}\right\|^{2}+\left\|p_{2}\right\|^{2}
\end{aligned}
$$

We then have that

$$
\begin{aligned}
\left(p_{h}, \operatorname{div} w_{h}\right) & =\left(p_{1}, \operatorname{div} w_{h}\right)+\left(p_{2} \operatorname{div} w_{h}\right) \\
& \geqslant \gamma_{1}^{2}\left\|p_{1}\right\|^{2}-\left\|\operatorname{div} w_{h}\right\|\left\|p_{2}\right\| \\
& \geqslant \gamma_{1}^{2}\left\|p_{1}\right\|^{2}-C\left\|p_{1}\right\|\left\|p_{2}\right\| \\
& \geqslant \frac{\gamma_{1}^{2}}{2}\left\|p_{1}\right\|^{2}-\frac{C}{2 \gamma_{1}^{2}}\left\|p_{2}\right\|^{2} \\
& \geqslant \frac{\gamma_{1}^{2}}{2}\left\|p_{h}\right\|^{2}-c_{1} s\left(p_{h}, p_{h}\right)
\end{aligned}
$$

and the statement of the Lemma follows.

We finally have the following theorem.

Theorem 6

Let $\alpha$ be big enough. Then there exists $\Gamma>0$ such that for given $\left(v_{h}, p_{h}\right) \in V_{h} \times Q_{h}$ there exists $\left(w_{h}, q_{h}\right) \in V_{h} \times Q_{h}$ with $\left\|\left(w_{h}, q_{h}\right)\right\| \leqslant 1$ and

$$
a\left(\left(v_{h}, p_{h}\right),\left(w_{h}, q_{h}\right)\right)+\alpha s\left(p_{h}, q_{h}\right) \geqslant \Gamma\left\|\mid\left(v_{h}, p_{h}\right)\right\|
$$

Proof

We have that

$$
\begin{aligned}
a\left(\left(v_{h}, p_{h}\right),\left(-w_{h}, 0\right)\right) & =-\left(\nabla v_{h}, \nabla w_{h}\right)+\left(p_{h}, \operatorname{div} w_{h}\right) \\
& \geqslant-\left\|\nabla v_{h}\right\|\left\|\nabla w_{h}\right\|+\gamma^{2}\left\|p_{h}\right\|-\alpha s\left(p_{h}, p_{h}\right) \\
& \geqslant-\frac{1}{2 \varepsilon}\left\|\nabla v_{h}\right\|^{2}-\frac{\varepsilon}{2}\left\|\nabla w_{h}\right\|^{2}+\gamma^{2}\left\|p_{h}\right\|^{2}-\alpha s\left(p_{h}, p_{h}\right) \\
& \geqslant\left(\gamma^{2}-C \varepsilon / 2\right)\left\|p_{h}\right\|^{2}-\frac{1}{2 \varepsilon}\left\|\nabla v_{h}\right\|^{2}-(C+\alpha) s\left(p_{h}, p_{h}\right)
\end{aligned}
$$


Choosing $\varepsilon$ and $\theta$ sufficiently small we finally obtain

$$
a\left(\left(v_{h}, p_{h}\right),\left(v_{h}-\theta w_{h}, p_{h}\right)\right) \geqslant C\left(\left\|p_{h}\right\|^{2}+s\left(p_{h}, p_{h}\right)+\left\|\nabla v_{h}\right\|^{2}\right)
$$

which together with the estimate $\left\|\left(v_{h}-\theta w_{h}, p_{h}\right)\right\| \leqslant C \|\left|\left(v_{h}, p_{h}\right)\right| \mid$ concludes the proof.

We also have the standard estimate:

\section{Theorem 7}

There is a constant $C$ such that if $v \in H^{3}(\Omega)$ and $p \in H^{2}(\Omega)$ we have the following standard error estimate:

$$
\left\|p-p_{h}\right\|+\left\|\nabla\left(v-v_{h}\right)\right\| \leqslant C h^{2}
$$

The proof of this result follows the same lines as that of Theorem 3.

\section{NUMERICAL EXAMPLES}

We consider a problem with exact solution $u=\left(20 x y^{3}, 5 x^{4}-5 y^{4}\right), p=60 y x^{2}-20 y^{3}-5$. We use the exact values of $u$ in the nodes as Dirichlet data for the discrete problem. In Figure 1 we show the start meshes using the linear and the quadratic approximations. The successive refinement is accomplished by performing the longest edge bisection twice.

In Table I we show the convergence obtained using $P^{1}$ approximations with $\alpha=\frac{1}{2}$, and in Table II we give the convergence for the $P^{2}$-approximation with $\alpha=\frac{1}{4}$.

We remark that the observed convergence of the pressure is one half power of $h$ better than our estimates. This well-known behavior is discussed and analyzed (on quadrilateral meshes) in [5] using a similar stabilization method.

Finally, in Figures 2 and 3 we show the effect on the error of varying $\alpha$ on a fixed mesh for the $P^{1}-P^{1}$ and $P^{2}-P^{2}$ approximations, respectively. We note that the $P^{1}$ method is much

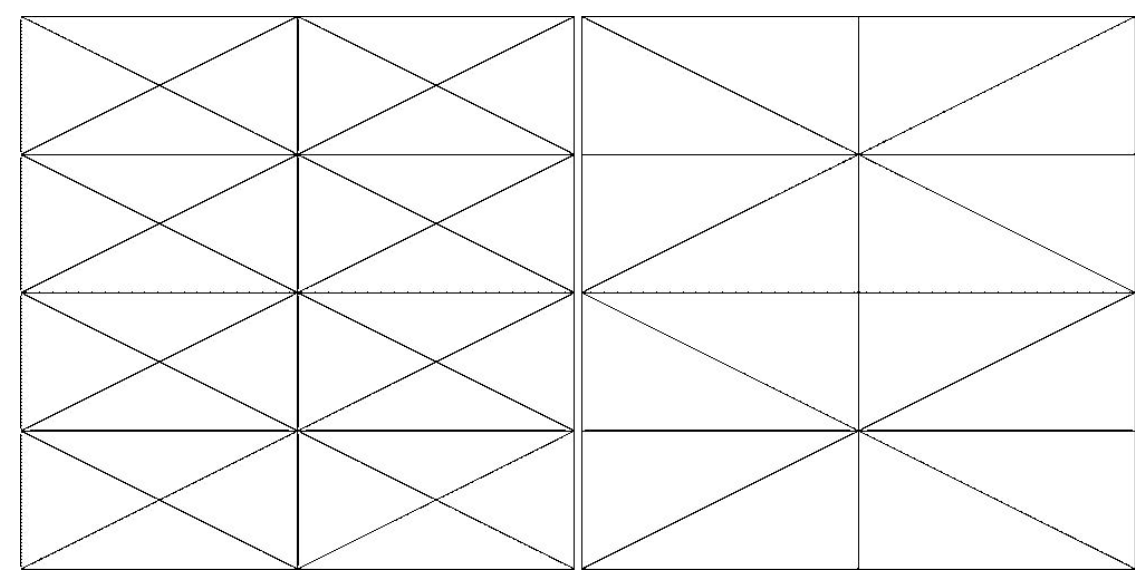

Figure 1. Start mesh for the $P^{1}$ case (left) and the $P^{2}$ case (right). 
Table I. Convergence for the $P^{1}$ case. The rates are based on the current and preceding values.

\begin{tabular}{lcccc}
\hline & \multicolumn{4}{c}{ Convergence in the $P^{1}$ case } \\
\cline { 2 - 5 }$h$ & $\left\|p-p_{h}\right\|$ & Rate & $\left\|\nabla\left(u-u_{h}\right)\right\|$ & Rate \\
\hline 0.25 & 6.0901 & - & 5.8183 & - \\
0.125 & 2.1793 & 1.4826 & 2.8804 & 1.0143 \\
0.0625 & 0.7188 & 1.6002 & 1.4090 & 1.0316 \\
0.03125 & 0.2303 & 1.6421 & 0.6953 & 1.0189 \\
\hline
\end{tabular}

Table II. Convergence for the $P^{2}$ case. The rates are based on the current and preceding values.

\begin{tabular}{lcccc}
\hline & \multicolumn{4}{c}{ Convergence in the $P^{2}$ case } \\
\cline { 2 - 5 }$h$ & $\left\|p-p_{h}\right\|$ & Rate & $\left\|\nabla\left(u-u_{h}\right)\right\|$ & Rate \\
\hline 0.3536 & 2.2780 & - & 1.3619 & - \\
0.1768 & 0.4271 & 2.4151 & 0.3357 & 2.0205 \\
0.0884 & 0.0790 & 2.4345 & 0.0828 & 2.0191 \\
0.0442 & 0.0153 & 2.3694 & 0.0205 & 2.0113 \\
\hline
\end{tabular}

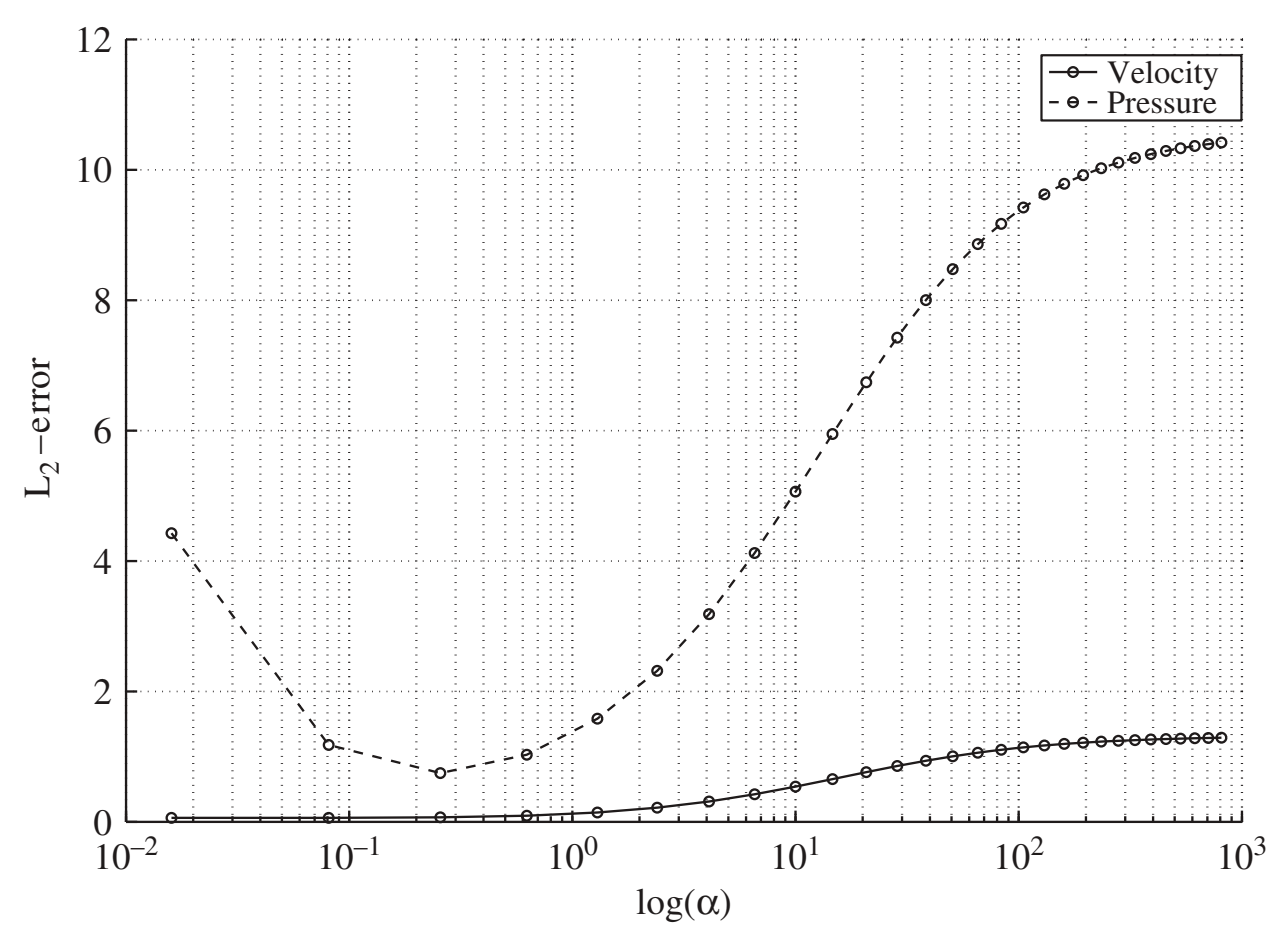

Figure 2. Effect of varying $\alpha$ for the $P^{1}$ case. 


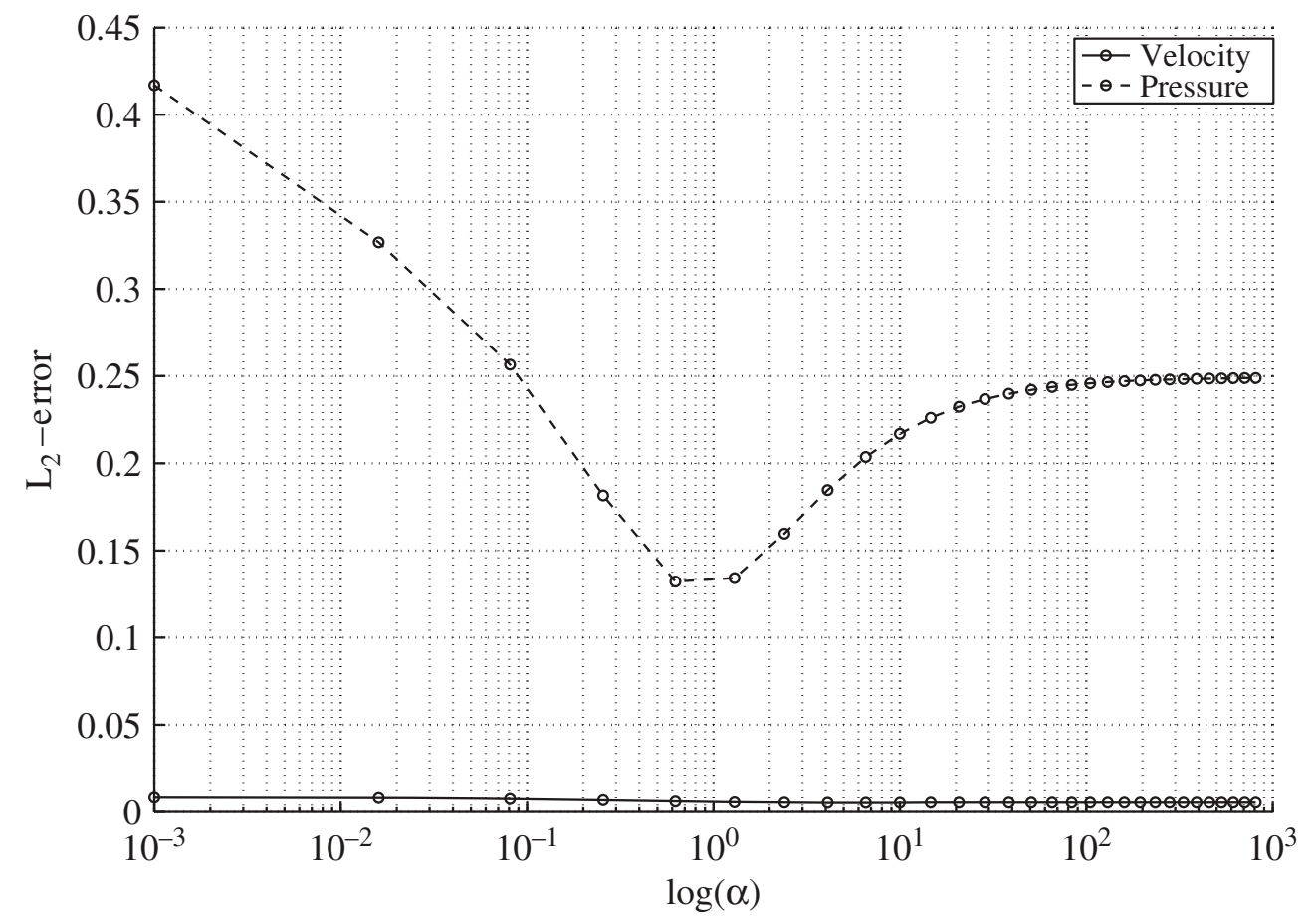

Figure 3. Effect of varying $\alpha$ for the $P^{2}$ case.

more sensitive with regard to the size of the parameter. The robustness of the $P^{2}-P^{2}$ method in this respect is related to the fact that the stabilization vanishes for the $P^{1}$ part of the approximation, indicating that the solution is forced toward the $P^{2}-P^{1}$ Taylor-Hood approximation as $\alpha$ increases.

\section{REFERENCES}

1. Girault V, Raviart PA. Finite Elements for the Navier-Stokes Equations. Springer: Berlin, 1986.

2. Brezzi F, Pitkäranta J. On the stabilization of finite element approximations of the Stokes equations. In Notes on Numerical Fluid Mechanics, Efficient Solutions of Elliptic Systems, Hackbusch W (ed.), vol. 10. Vieweg: Braunschweig, 1984.

3. Hughes TJR, Franca LP, Balestra M. A new finite element formulation for computational fluid dynamics: V. Circumventing the Babuska-Brezzi condition: a stable Petrov-Galerkin formulation for the Stokes problem accommodating equal order interpolation. Computer Methods in Applied Mechanics and Engineering 1986; 59:89-99.

4. Codina R, Blasco J. A finite element formulation for the Stokes problem allowing equal velocity-pressure interpolation. Computer Methods in Applied Mechanics and Engineering 1997; 143:373-391.

5. Becker R, Braack M. A finite element pressure gradient stabilization for the Stokes equations based on local projections. Calcolo 2001; 38(4):173-199.

6. Burman E, Hansbo P. Edge stabilization for the generalized Stokes problem: a continuous interior penalty method. Computer Methods in Applied Mechanics and Engineering 2006; 195:2393-2410.

7. Dohrmann C, Bochev P. A stabilized finite element method for the Stokes problem based on polynomial pressure projections. International Journal for Numerical Methods in Fluids 2004; 46:183-201.

8. Löhner R, Morgan K, Peraire J, Vahdati M. Finite element flux-corrected transport (FEM-FCT) for the Euler and Navier-Stokes equations. International Journal for Numerical Methods in Fluids 1987; 7:1093-1109.

9. Li J, He Y. A stabilized finite element method based on two local Gauss integrations for the Stokes equations. Journal of Computational and Applied Mathematics 2007; DOI: 10.1016/j.cam.2007.02.015.

10. Brezzi F, Fortin M. Mixed and Hybrid Finite Element Methods. Springer: New York, 1991. 